TITLE:

\title{
Uptake of adenine by purine permeases of Coffea canephora
}

$\operatorname{AUTHOR}(S)$ :

Kakegawa, Hirofumi; Shitan, Nobukazu; Kusano, Hiroaki; Ogita, Shinjiro; Yazaki, Kazufumi; Sugiyama, Akifumi

\section{CITATION:}

Kakegawa, Hirofumi ...[et al]. Uptake of adenine by purine permeases of Coffea canephora. Bioscience, biotechnology, and biochemistry 2019, 83(7): 1300-1305

\section{ISSUE DATE:}

2019

URL:

http://hdl.handle.net/2433/245789

\section{RIGHT:}

This is an Accepted Manuscript of an article published by Taylor \& Francis in Bioscience, biotechnology, and biochemistry on 18 April 2019, available online: http://www.tandfonline.com/10.1080/09168451.2019.1606698.; The fulltext file will be made open to the public on 18 April 2020 in accordance with publisher's 'Terms and Conditions for SelfArchiving'.; This is not the published version. Please cite only the published version.; この論文は出版社版でありません

。引用の際には出版社版をご確認ご利用ください。 
1 Uptake of adenine by purine permeases of Coffea canephora

2

$3 \quad{ }^{1}$ Hirofumi Kakegawa, ${ }^{2}$ Nobukazu Shitan, ${ }^{1}$ Hiroaki Kusano, ${ }^{3}$ Shinjiro Ogita, ${ }^{1}$ Kazufumi

4 Yazaki, ${ }^{1 *}$ Akifumi Sugiyama

5

$6 \quad{ }^{1}$ Research Institute for Sustainable Humanosphere, Kyoto University, Gokasho, Uji,

7 611-0011, Japan, ${ }^{2}$ Laboratory of Medicinal Cell Biology, Kobe Pharmaceutical

8 University, Kobe, 658-0003, Japan. ${ }^{3}$ Faculty of Life and Environmental Sciences,

9 Prefectural University of Hiroshima, 5562 Nanatsukacho, Shobara, Hiroshima, 10 727-0023, Japan.

11

12

13 *Corresponding author: Akifumi Sugiyama, tel: +81-774-38-3618, fax:

14 +81-774-38-3623, e-mail: akifumi_sugiyama@rish.kyoto-u.ac.jp 


\section{Abstract}

17 Purine permeases (PUPs) mediate the proton-coupled uptake of nucleotide bases and

18 their derivatives into cytosol. PUPs facilitate uptake of adenine, cytokinins and nicotine.

19 Caffeine, a purine alkaloid derived from xanthosine, occurs in only a few eudicot species, including coffee, cacao, and tea. Although caffeine is not an endogenous metabolite in Arabidopsis and rice, AtPUP1 and OsPUP7 were suggested to transport caffeine. In this study, we identified 15 PUPs in the genome of Coffea canephora. Direct uptake measurements in yeast demonstrated that CcPUP1 and CcPUP5 facilitate adenine-but not caffeine-transport. Adenine uptake was $\mathrm{pH}$-dependent, with increased activity at pH 3 and 4, and inhibited by nigericin, a potassium-proton ionophore, suggesting that CcPUP1 and CcPUP5 function as proton-symporters. Furthermore, adenine uptake was not competitively inhibited by an excess amount of caffeine, which implies that PUPs of $C$. canephora have evolved to become caffeine-insensitive to promote efficient uptake of adenine into cytosol.

Keywords; adenine, caffeine, Coffea canephora, purine permease 


\section{Introduction}

Purine permeases (PUPs) constitute a large family of transporter molecules that are localized at the plasma membrane in plants, where they facilitate proton-coupled uptake of nucleotide bases and their derivatives, including adenine, cytokinins, and nicotine [1,2]. The first PUP member, AtPUP1 of Arabidopsis thaliana, was identified as the gene that complemented a yeast mutant in adenine uptake [3]. The protonophore carbonyl cyanide $m$-chlorophenyl hydrazine inhibits the adenine uptake activity of AtPUP1, suggesting that this PUP functions as a proton symporter [4]. In addition, the adenine uptake activity of AtPUP1 is competitively inhibited by purine derivatives including cytokinins and caffeine [4], thus suggesting that AtPUP1 mediates the uptake of a broad range of substrates.

Although Arabidopsis contains 23 PUP members in its genome, only four PUP genes have been analyzed to date. Of these, both AtPUP1 and AtPUP2 have similar substrate specificity for the uptake of adenine and cytokinins, but their tissue-specific expression patterns differ: AtPUP1 is expressed mainly in leaf hydathode tissue and the stigmatic surface, whereas AtPUP2 in expressed predominantly in vascular tissues [4,5]. Furthermore, AtPUP1 reportedly also facilitates the uptake of pyridoxine [6].

Another of the AtPUPs characterized to date, AtPUP3, is expressed in pollen, but the protein did not demonstrate any transport activity in a yeast system [4]. Through its cytokinin uptake activity, AtPUP14 is involved in the spatiotemporal distribution of cytokinin in the meristem and thus in plant morphogenesis [7]. Rice (Oryza sativa) contains 12 PUP members in its genome, but only one member, OsPUP7, was characterized as being involved in plant growth and development, possibly mediating cytokinin transport [8]. Although direct transport activity was not measured, OsPUP7 conferred sensitivity to caffeine in yeast, suggesting that OsPUP7 may take up the caffeine [8].

In addition to adenine and cytokinins, derivatives of nucleotide bases include the alkaloids nicotine and caffeine. The ability of a PUP to take up nicotine was first identified in Nicotiana tabacum [9,10], in which NtNUP1 acquires nicotine from the apoplast, particularly in root tips. The suppression of NtNUP1 expression in tobacco hairy roots decreased the nicotine content in the tissue [10], and measurement of direct 
uptake activity in yeast showed that NtNUP1 is an uptake transporter of - in addition to nicotine-pyridoxamine, pyridoxine, and anatabine [9]. Although the results of competitive inhibition and yeast growth assays have suggested that PUPs in Arabidopsis, and rice potentially transport caffeine $[3,4,8]$, their caffeine uptake activity has not been measured directly, nor have the PUPs in any caffeine-synthesizing species been characterized. Caffeine is synthesized by only a few eudicot plants, such as coffee (Coffea spp.), cacao (Theobroma cacao), and tea (Camellia sinensis) [11,12]. Coffee is an important cash crop and is cultivated across more than 11 million hectares [13]. Two species (C. arabica and C. canephora) account for nearly all coffee bean production. $C$. arabica is an autogamous allotetraploid species originating from a cross between $C$. canephora and C. eugenioides [14]. In this report we identified 15 PUP members from C. canephora, and characterized the transport activity of CcPUPs in yeast.

\section{Materials and Methods}

Chemicals

Chemicals were obtained from Wako Pure Chemical Industries (Osaka, Japan) or Nacalai Tesque (Kyoto, Japan), unless otherwise stated.

\section{Identification and sequence analysis of PUP homologs in C. canephora}

We used the predicted amino acid sequence of AtPUP1 as a query sequence in a BLASTP search of the publicly available database Coffee Genome Hub (http://coffee-genome.org/) to find the PUP genes in C. canephora. Using default search parameters, we identified 15 CcPUPs, which we named CcPUP1 to CcPUP15 in order of their loci in the genome.

\section{Construction of plasmids and transformation of yeast}

The coding sequences of CCPUP1 and CcPUP5 were amplified in $25-\mu \mathrm{L}$ reaction mixtures containing cDNA of cultured C. canephora cells, $0.5 \mu \mathrm{L}$ of PrimeSTAR GXL DNA Polymerase (Takara, Japan), $5 \mu \mathrm{L}$ of $5 \times$ PrimeSTAR GXL buffer, $16 \mu \mathrm{L}$ of distilled water, $2 \mu \mathrm{L}$ of dNTPs (2.5 mM), and 5 pmol of each appropriate primer (CcPUP1, 5'-CACCATGCCAGTCAATGAGGAACC-3' and 
5'-TCAGCACAACGAGTCATTAGTAG-3'; CcPUP5, 5'-CACCATGGAGAATACTACTCAAGAAATGG-3' and 5'-TCAAGAAGTCCCTAGGAAAGAA-3'). PCR amplification conditions were: denaturation at $98^{\circ} \mathrm{C}$ for $1 \mathrm{~min}$; 30 cycles of $98^{\circ} \mathrm{C}$ for $10 \mathrm{~s}, 60{ }^{\circ} \mathrm{C}$ for $15 \mathrm{~s}$, and $68^{\circ} \mathrm{C}$ for $1 \mathrm{~min}$. A final extension was conducted for 5 minutes at $58^{\circ} \mathrm{C}$. PCR amplicons were purified by using the Wizard SV Gel and PCR Clean-Up System (Promega, San Luis Obispo, CA) according to the manufacturer's protocol. CcPUP1 and CcPUP5 cDNAs were ligated into pENTR/D-TOPO vector (Invitrogen, Carlsbad, CA). DNA sequence of CcPUP7 was synthesized by Genewiz (Kawaguchi, Japan), and ligated into pENTR/D-TOPO. These were then transferred into pYES-DEST52 (Invitrogen) by using Gateway cloning technology (Invitrogen) according to the manufacturer's instructions. The cDNA-carrying pYES-DEST52 vectors were used to transduce an

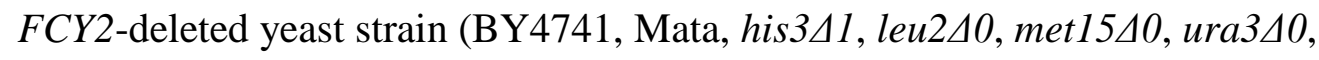
YER056C::kanMX4; Invitrogen) according to the manufacturer's protocol; pYES-DEST52 lacking a CcPUP cDNA insert was used as a negative control.

\section{Growth assay}

Gene expression in pYES-DEST52-transduced yeast cells can be induced by galactose and repressed by glucose. The basic (control) medium for the growth assays was minimal medium (lacking uracil and glucose); $2 \%$ galactose and 1\% raffinose were added as carbon sources to induce gene expression. Basic medium containing $2 \%$ glucose was used to repress gene expression. Transduced yeast cells were pre-incubated in the inducing medium or repression medium for 2 days at $30^{\circ} \mathrm{C}$, and then were diluted to an $\mathrm{OD}_{600}$ of 0.002 by adding sterile water. Then $40 \mu \mathrm{l}$ of each diluted solution was applied to an agar plate containing caffeine, which was incubated at $30{ }^{\circ} \mathrm{C}$ for 5 days, according to the literatures $[4,8]$.

\section{Measurement of transport of purine derivatives in yeast}

For caffeine uptake assays, transduced yeast cells were pre-incubated in repression medium for 2 days at $30{ }^{\circ} \mathrm{C}$. Cells were harvested by centrifugation, washed, and resuspended in inducing medium to a final $\mathrm{OD}_{600}$ of 0.2. Yeast cells were incubated at 
$30{ }^{\circ} \mathrm{C}$ for 40 hours and then harvested, washed, and resuspended in $100 \mathrm{mM}$ sodium citrate buffer ( $\mathrm{pH} 3.0$ ) containing $1 \%$ glucose to a final $\mathrm{OD}_{600}$ of 12 . Before initiation of the transport assay, yeast cells $(100 \mu \mathrm{L})$ were preincubated at $30{ }^{\circ} \mathrm{C}$ for $2 \mathrm{~min}$; then assay buffer $(105 \mu \mathrm{L})$ containing $100 \mathrm{mM}$ citrate buffer, $\mathrm{pH}$ 3.0, 1\% glucose, $176 \mathrm{~Bq}$ $\mu \mathrm{L}^{-1}{ }^{14} \mathrm{C}$-labeled caffeine (American Radiolabeled Chemicals, St Louis, MO) and unlabeled caffeine was added. A 40- $\mu \mathrm{L}$ sample was withdrawn from the reaction tube after 30, 60, 120, and $180 \mathrm{~s}$; each sample was transferred to $4 \mathrm{~mL}$ of ice-cold water, filtered onto glass-fiber filters (GE Healthcare, Chicago, IL), and then washed with 4 mL of water in a manifold (Merck Millipore, Burlington, MA). For adenine uptake assays, the incubation was initiated by adding $103 \mu \mathrm{L}$ of assay buffer containing 100 mM citrate buffer, $\mathrm{pH}$ 3.0, 1\% glucose, $718 \mathrm{~Bq} \mu \mathrm{L}^{-1}{ }^{3} \mathrm{H}$-labeled adenine (Moravek Biochemicals, Brea, CA) and unlabeled adenine at the final concentration of $100 \mu \mathrm{M}$, according to the literature [4].

To vary the $\mathrm{pH}$ of the assay, yeast cells were grown at $30{ }^{\circ} \mathrm{C}$ for 40 hours, washed, and resuspended in $100 \mathrm{mM}$ sodium citrate buffer for which the $\mathrm{pH}$ was adjusted. For assays involving nigericin, a reaction buffer containing $4.0 \mathrm{mM}$ nigericin was used. For competitive inhibition assays, $94 \mu \mathrm{L}$ of buffer containing $540 \mu \mathrm{M}$ caffeine, adenine, or sucrose was added 2 min before the start of the reaction, after preincubation, reactions were initiated by adding $9 \mu \mathrm{L}$ of buffer containing $8.2 \mathrm{kBq} \mu \mathrm{L}^{-1}$ ${ }^{3}$ H-labeled adenine.

\section{Results}

150 Identification and expression analysis of purine permeases of $C$. canephora

151 In this study, we used AtPUP1 as a query in a BLASTP search of the genomic sequence of C. canephora in the public database Coffee Genome Hub (http://coffee-genome.org/) to identify PUPs in this species. We named the 15 PUPs that we obtained as CcPUP1 through CcPUP15, in order of their genomic loci, as follows: CcPUP1, Cc02g25680; CcPUP2, Cc03g11350; CcPUP3, Cc03g13540; CcPUP4, Cc06g15040; CcPUP5,

156 Cc08g01780; CcPUP6, Cc08g11780; CcPUP7, Cc09g04610; CcPUP8, Cc09g08430;

157 CcPUP9, Cc09g09080; CcPUP10, Cc09g09090; CcPUP11, Cc09g09160; CcPUP12, 
159 A phylogenetic tree constructed by using the amino acid sequences of CcPUPs and

160 characterized PUPs from Arabidopsis, rice, and tobacco showed that AtPUP1 and

161 OsPUP7, which were 34\% homologous at the amino acid level, clustered in different clades (Fig. 1A) even though both proteins have been suggested to transport caffeine.

We then used the RNA-seq data available in Coffee Genome Hub (http://coffee-genome.org/) to summarize the tissue expression of CCPUPs (Fig. 1B). Whereas CcPUP1, CcPUP4, CcPUP6, and CcPUP12 are expressed in most tissues, CcPUP2 is expressed more specifically in leaves and roots. In addition, CcPUP7 is strongly expressed in perisperm and endosperm, where caffeine is highly accumulated.

CcPUP1 and CCPUP5 confer sensitivity to caffeine in yeast

Results of yeast sensitivity tests suggest that PUPs transport caffeine $[4,8]$. We therefore individually cloned CcPUP cDNAs into the pYES-DEST52 vector, in which gene expression is regulated by the GAL1 promoter, and used the plasmids to transform yeast mutant fcy2, which is deficient in adenine uptake [3]. Yeast transformants expressing CcPUP1, CcPUP4, CcPUP5, CcPUP6, CcPUP7, and CcPUP12 were cultured. Compared with that of the vector control, growth of the yeast transformants expressing CcPUP1 and CCPUP5 was suppressed on induction medium containing $0.2 \%$ caffeine (Fig. 2). No difference in growth was observed for yeast transformants harboring CcPUP4, CcPUP6, CcPUP7, or CcPUP12 (Supplementary Fig. 1)

Transport assays

181 Because the results of the yeast growth assay suggested that CcPUP1 and CcPUP5 have caffeine uptake activity, we analyzed their direct transport activity by determining the caffeine contents in yeast transformants after their incubation on caffeine as a substrate. No uptake activity was observed for transformants expressing either CcPUP1 or CcPUP5 (Fig. 3A). When the $\mathrm{pH}$ condition was modified from $\mathrm{pH} 3$ to 7, there was no caffeine uptake activity in these cells (Supplementary Fig. 2). The uptake activity for adenine was then measured using the same transformants. Higher amount of adenine was transported in yeast transformants expressing CcPUP1 or CcPUP5 than the vector control (Fig. 3B). In addition, the adenine uptake activity of CcPUP1 and CcPUP5 was 
190 higher at a pH of 3 or 4 and decreased when the pH was increased (Fig. 4).

Because CcPUP7 is highly expressed in the perisperm and endosperm, where caffeine is highly accumulated, the caffeine and adenine uptake were also tested for CcPUP7. The uptake activity was not observed in yeast transformants (Supplementary Fig. 3).

Inhibition of adenine uptake

To analyze whether adenine uptake by CcPUP1 and CcPUP5 utilizes a proton gradient, we measured adenine uptake in the presence of nigericin, an ionophore that exchanges $\mathrm{K}^{+}$for $\mathrm{H}^{+}$across membranes and thus abolishes a $\mathrm{pH}$ gradient. Adenine uptake by CcPUP1 and CcPUP5 was decreased by about $42 \%$ and 51\%, respectively, in the presence of nigericin compared with control values (Fig. 5). Nigericin also reduced the adenine uptake of AtPUP1 (Fig. 5C).

Caffeine was suggested to competitively inhibit the ability of AtPUP1 to take up adenine [3]. To investigate whether caffeine competitively inhibits adenine uptake by CcPUP1 and CcPUP5, we conducted a transport assay under which the incubation media contained 10-fold more caffeine than adenine; controls for this assay included 10-fold increased amounts of adenine and sucrose. For both CcPUP1 and CcPUP5, the uptake of radioactive adenine was decreased in the presence of excess adenine but was unaffected under conditions of excess caffeine or sucrose (Fig. 5). In contrast to findings for CcPUP1 and CcPUP5, addition of an excess of caffeine diminished the uptake of adenine by AtPUP1 (Fig. 5F), in line with a previous report [3].

\section{Discussion}

214 Purine bases such as adenine and guanine are ubiquitous metabolites that are found in

215 all organisms. In addition to purine nucleotides, several plants synthesize purine

216 alkaloids, including caffeine and theobromine [15]. The caffeine synthesized by Coffea

217 spp. accumulates predominantly in seeds and leaves, where caffeine restricts

218 development and growth of other organisms [16] and also stimulates plant defense

219 response by affecting signaling pathways [17,18]. In addition, caffeine is secreted from

220 the roots during germination, when it is thought to modulate interactions with pathogens 221 and mycoparasites [19,20]. 
Several lines of evidence support the importance of membrane transport in the function of metabolites [21,22], and various families of transporters for nucleotide bases and their derivatives have been characterized [1,23]. In the current study, we analyzed the purine permease family members, which are uptake transporters for various purine bases and their derivatives [2], in C. canephora, for which genomic and transcriptomic data are publicly available $[11,24]$. Among the 15 PUP genes that we discovered in the genome of $C$. canephora, two (CcPUP1 and CcPUP5) were identified as encoding candidate uptake transporters, according to growth assays using caffeine-containing media. Using radioactive substrates in a yeast-based system, we showed that both CcPUP1 and CcPUP5 uptake adenine, possibly in a proton-symport manner. Even though growth assay results suggested that both CcPUP1 and CcPUP5 can uptake caffeine, neither transporter recognized caffeine as a substrate. The apparent sensitivity of CcPUP1 and CcPUP5 transformants to caffeine might merely reflect the growth retardation of yeast expressing a membrane transporter. Even in liquid media without caffeine, the growth of the CcPUP1 and CcPUP5 transformants was suppressed compared with vector controls (Supplementary Fig. 4).

In conclusion, among the 15 PUPs that we identified in C. canephora, we found that CcPUP1 and CcPUP5, which are adenine transporters, not inhibited by caffeine. The insensitivity of CcPUPs to caffeine may be physiologically important in $C$. canephora, where these proteins need to distinguish adenine from caffeine to efficiently take up adenine in various cells. We surmise that purine permeases in C. canephora have evolved to differentiate adenine from caffeine.

Acknowledgements

246 We thank Ms. Shuka Nagayama for technical assistance in maintaining tissue cultures.

\section{Author Contributions}

249 HK, NS, SO, KY, and AS conceived and designed the experiments; HK and AS

250 performed the experiments; HK, NS, HK*, KY, and AS analyzed the data; and HK and

251 AS wrote the paper with input from all coauthors. 


\section{Disclosure Statement}

255 No potential conflict of interest was reported by the authors.

256

257 Funding

258 This study was supported in part by grants from JSPS KAKENHI (16K14901 and $25918 \mathrm{H} 02313$ to AS) and from the Research Institute for Sustainable Humanosphere 260 (Mission research and Mission 5-1) and by the Research Unit for Development of 261 Global Sustainability, Kyoto University. 


\section{Figure Legends}

265 Figure 1. Phylogenetic analysis of purine permease (PUP) proteins in Arabidopsis (At),

266 rice (Os) and Coffea canephora (Cc) and their gene expression levels. (a) A

267 phylogenetic tree was generated by using MEGA 7.0 software [25]. The amino acid

268 sequences of PUPs were aligned by using the MUSCLE program. The maximum

269 likelihood method was used to construct a phylogenetic tree with 1000 bootstrap

270 replications. Bootstrap values (maximum, 100) are shown at nodes generating clades.

271 (b) Heat map according to the number of reads per kilobase per million mapped reads

272 (RPKM) for each gene.

273

274 Figure 2. Growth of CcPUP-transformed yeast on media containing $0.2 \%$ caffeine after

275 incubation at $30{ }^{\circ} \mathrm{C}$ for 5 days.

Figure 3. Transport assay using CcPUP1 and CcPUP5-expressing yeast. (a)

278 Time-dependent uptake of caffeine in yeast expressing CcPUP1 and CcPUP5. The final concentration of caffeine was $100 \mu \mathrm{M}$. (b) Time-dependent uptake of adenine in yeast transformants expressing CcPUP1 and CcPUP5. The final concentration of adenine was $100 \mu \mathrm{M}$. Data are presented as mean \pm SD $(\mathrm{n}=3)$; different letters indicate values that are significantly different $(P<0.05)$ according to Tukey’s Honestly Significant Difference test.

Figure 4. Effect of pH on adenine uptake by CcPUP1 and CcPUP5. Adenine uptake was determined after 3 min of incubation in sodium citrate buffer; the final concentration of adenine was $100 \mu \mathrm{M}$. Data are presented as mean \pm SD of three replicates; different letters indicated values that are significantly different $(P<0.05)$ according to Tukey's Honestly Significant Difference test.

Figure 5. Inhibition of adenine uptake. Adenine uptake by (a) CcPUP1, (b) CcPUP5, and (b) AtPUP1 after 3 min of incubation in the presence of nigericin. Adenine uptake by (d) CcPUP1, (e) CcPUP5, and (f) AtPUP1 after 3 min of incubation in the presence of excess amounts of adenine, caffeine, and sucrose. The final concentration of each 
substrate was $25 \mu \mathrm{M}$. Data are given as mean \pm SD $(n=3)$; different letters indicate values that are significantly different $(P<0.05)$ according to the Student t-Test $(\mathrm{A}-\mathrm{C})$ or Tukey’s Honestly Significant Difference test (D-F).

Supplementary Figure 1. Growth of CcPUP-expressing yeast on media containing caffeine after incubation at $30^{\circ} \mathrm{C}$ for 5 days.

Supplementary Figure 2. Effect of pH on caffeine uptake by CcPUPs. Caffeine uptake was determined after 3 min of incubation in sodium citrate buffer. The final concentration of caffeine was $100 \mu \mathrm{M}$. Data are presented as mean \pm SD of three replicates.

Supplementary Figure 3. Transport assay using CcPUP7-expressing yeast. (a) Uptake of caffeine in yeast expressing CcPUP7. The final concentration of caffeine was $100 \mu \mathrm{M}$.

(b) Uptake of adenine in yeast transformants expressing CcPUP7. The final concentration of adenine was $100 \mu \mathrm{M}$. Data are presented as mean $\pm \mathrm{SD}$ of three replicates.

Supplementary Figure 4. Growth of yeast in liquid media. The $\mathrm{OD}_{600}$ of each culture was measured over 24 hours. Data are presented as mean \pm SD of three replicates.

\section{References}

[1] Shitan N, Kato K, and Shoji T. Alkaloid transporters in plants. Plant Biotechnology. 2014;31(5):453-463.

[2] Jelesko JG. An expanding role for purine uptake permease-like transporters in plant secondary metabolism. Frontiers in Plant Science. 2012;3:78.

[3] Gillissen B, Burkle L, Andre B, Kuhn C, Rentsch D, Brandl B, and Frommer WB. A new family of high-affinity transporters for adenine, cytosine, and purine derivatives in arabidopsis. Plant Cell. 2000;12:291-300.

[4] Burkle L, Cedzich A, Dopke C, Stransky H, Okumoto S, Gillissen B, Kuhn C, and Frommer WB. Transport of cytokinins mediated by purine transporters of the PUP family expressed in phloem, hydathodes, and pollen of Arabidopsis. Plant J. 2003;34:13-26. 
[5] Cedzich A, Stransky H, Schulz B, and Frommer WB. Characterization of Cytokinin and Adenine Transport in Arabidopsis Cell Cultures. Plant Physiology. 2008;148:1857-1867.

[6] Szydlowski N, Buerkle L, Pourcel L, Moulin M, Stolz J, and Fitzpatrick TB. Recycling of pyridoxine (vitamin B6) by PUP1 in Arabidopsis. Plant J. 2013;75:40-52.

[7] Zurcher E, Liu JC, di Donato M, Geisler M, and Muller B. Plant development regulated by cytokinin sinks. Science. 2016;353:1027-1030.

[8] Qi ZY, and Xiong LZ. Characterization of a Purine Permease Family Gene OsPUP7 Involved in Growth and Development Control in Rice. J Integrative Plant Biology. 2013;55:1119-1135.

[9] Kato K, Shitan N, Shoji T, and Hashimoto T. Tobacco NUP1 transports both tobacco alkaloids and vitamin B6. Phytochemistry. 2015;113:33-40.

[10] Hildreth SB, Gehman EA, Yang HB, Lu RH, Ritesh KC, Harich KC, Yu S, Lin JS, Sandoe JL, Okumoto S, Murphy AS, and Jelesko JG. Tobacco nicotine uptake permease (NUP1) affects alkaloid metabolism. Proceedings of the National Academy of Sciences of the United States of America. 2011;108:18179-18184.

[11] Denoeud F, Carretero-Paulet L, Dereeper A, Droc G, Guyot R, Pietrella M, Zheng CF, Alberti A, Anthony F, Aprea G, Aury JM, Bento P, Bernard M, Bocs S, Campa C, Cenci A, Combes MC, Crouzillat D, Da Silva C, Daddiego L, De Bellis F, Dussert S, Garsmeur O, Gayraud T, Guignon V, Jahn K, Jamilloux V, Joet T, Labadie K, Lan TY, Leclercq J, Lepelley M, Leroy T, Li LT, Librado P, Lopez L, Munoz A, Noel B, Pallavicini A, Perrotta G, Poncet V, Pot D, Priyono, Rigoreau M, Rouard M, Rozas J, Tranchant-Dubreuil C, VanBuren R, Zhang Q, Andrade AC, Argout X, Bertrand B, de Kochko A, Graziosi G, Henry RJ, Jayarama, Ming R, Nagai C, Rounsley S, Sankoff D, Giuliano G, Albert VA, Wincker $\mathrm{P}$, and Lashermes $\mathrm{P}$. The coffee genome provides insight into the convergent evolution of caffeine biosynthesis. Science. 2014;345:1181-1184.

[12] Xia E-H, Zhang H-B, Sheng J, Li K, Zhang Q-J, Kim C, Zhang Y, Liu Y, Zhu T, Li W, Huang H, Tong Y, Nan H, Shi C, Shi C, Jiang J-J, Mao S-Y, Jiao J-Y, Zhang D, Zhao Y, Zhao Y-J, Zhang L-P, Liu Y-L, Liu B-Y, Yu Y, Shao S-F, Ni D-J, Eichler EE, and Gao L-Z. The Tea Tree Genome Provides Insights into Tea Flavor and Independent Evolution of Caffeine Biosynthesis. Molecular Plant. 2017;10:866-877.

[13] Laderach P, Ramirez-Villegas J, Navarro-Racines C, Zelaya C, Martinez-Valle A, 
and Jarvis A. Climate change adaptation of coffee production in space and time. Climatic Change. 2017;141:47-62.

[14] Lashermes P, Combes MC, Robert J, Trouslot P, D'Hont A, Anthony F, and

[15] Ashihara H, Mizuno K, Yokota T, and Crozier A. Xanthine Alkaloids:

[16] Kim YS, and Sano H. Pathogen resistance of transgenic tobacco plants producing caffeine. Phytochemistry. 2008;69:882-888.

[17] Kim YS, Choi YE, and Sano H. Plant vaccination: Stimulation of defense system by caffeine production in planta. Plant Signaling \& Behavior. 2010;5:489-493.

[18] Sano H, Kim YS, and Choi YE. Like Cures Like: Caffeine Immunizes Plants Against Biotic Stresses. "New Light on Alkaloid Biosynthesis and Future Prospects," ed. GiglioliGuivarch N, pp. 273-300 (2013).

[19] Baumann TW, and Gabriel H. Metabolism and excretion of caffeine during germination of Coffea arabica L. Plant and Cell Physiology. 1984;25:1431-1436.

[20] Sugiyama A, Sano CM, Yazaki K, and Sano H. Caffeine fostering of mycoparasitic fungi against phytopathogens. Plant Signaling \& Behavior. 2016;11(1):e1113362.

[21] Larsen B, Xu DY, Halkier BA, and Nour-Eldin HH. Advances in methods for identification and characterization of plant transporter function. J Experimental Botany. 2017;68:4045-4056.

[22] Shitan N. Secondary metabolites in plants: transport and self-tolerance mechanisms. Bioscience Biotechnology and Biochemistry. 2016;80:1283-1293.

[23] Girke C, Daumann M, Niopek-Witz S, and Mohlmann T. Nucleobase and nucleoside transport and integration into plant metabolism. Frontiers in Plant Science. 2014;5:443.

[24] Mondego JMC, Vidal RO, Carazzolle MF, Tokuda EK, Parizzi LP, Costa GGL, Pereira LFP, Andrade AC, Colombo CA, Vieira LGE, Pereira GAG, and Brazilian Coffee Genome Project C. An EST-based analysis identifies new genes and reveals distinctive gene expression features of Coffea arabica and Coffea canephora. Bmc Plant Biology. 2011;11:30.

[25] Kumar S, Stecher G, and Tamura K. MEGA7: Molecular Evolutionary Genetics 

Analysis Version 7.0 for Bigger Datasets. Molecular Biology and Evolution.

401 2016;33:1870-1874.

402

403

404 